Pituitary enlargement on MRI in congenital hypothyroidism, mimicking a sellar or suprasellar tumor, is reported from the Bai Jerbai Wadia Hospital for Children and Institute of Child Health and Research Centre, Parel, Bombay 400 012, India. (Desia MP et al. Arch Pediatr Adolesc Med June 1996;150:623-628). The $\mathrm{CH}$ was long-standing and untreated. MRI was abnormal in all patients. One child presented with acute headache, vomiting, and papilledema. MRI showed pituitary enlargement, suggesting a pituitary adenoma. Bone age was retarded. Symptoms improved within 10 days of levothyroxine therapy, and an MRI at 2 months showed marked regression of pituitary size. Serum triiodothyronine and thyroxine levels were low, and thyrotropin was elevated in all patients. Prolactin was elevated in 5 patients and was highest in the child with neurologic symptoms. This complication of congenital hypothyroidism will be encountered primarily in developing countries and is rare in the US, since most patients will have received treatment soon after birth. However, an awareness of pituitary enlargement as a sign of $\mathrm{CH}$ will avoid unnecessary neurosurgical intervention in children with untreated or subtle hypothyroidism.

\title{
TOXIC DISORDERS
}

\section{COCAINE-INDUCED HORMONAL AND BEHAVIORAL CHANGES}

Behavioral and hormonal responses in 30 preterm cocaine-exposed infants were compared with a cohort of 30 non-cocaine-exposed preterm infants at the Touch Research Institute, University of Miami School of Medicine, FL. Cocaine-exposed infants had smaller head circumference at birth, longer stays in the intensive care unit, a higher incidence of intraventricular hemorrhage, inferior performance on the Brazelton Neonatal Behavioral Assessment Scale (range of state, regulation of state, and depression clusters), decreased periods of quiet sleep, and increased levels of agitated behavior, including tremulousness, limb movements, and clenched fists. They also had higher urinary norepinephrine, dopamine, and cortisol levels and lower plasma insulin levels than controls. Epinephrine and glucose levels were unchanged. (Scafidi FA, Field TM et al. Cocaine-exposed preterm nenates show behavioral and hormonal differences. Pediatrics June 1996;97:851-855). (Reprints: Dr Tiffany M Field, Touch Research Institute, University of Miami School of Medicine, PO Box 016820, Miami, FL 33101).

COMMENT. Cocaine-exposed infants require careful follow-up for early diagnosis and therapy of neurobehavioral complications. A frequent history of prenatal cocaine exposure in foster children with attention deficit hyperactivity disorders is of interest in relation to the changes in catecholamine metabolism noted in the above study. For reference to neurological correlates of fetal cocaine exposure, see Ped Neur Briefs Feb 1996;10:9-10; and Progress in Pediatric Neurology I and II PNB Publishers, 1991 and 1994.

Other prenatal toxic factors that may underly cognitive, behavioral, and attentional deficits in childhood include alcohol, PCBs, and nicotine.

Fetal alcohol syndrome and ophthalmological abnormalities are reported in 25 children examined at Children's Hospital, Goteberg, Sweden. (Stromland K, Hellstrom A. Pediatrics June 1996;97:845-850). The fundus was abnormal in 23, of whom 19 had optic nerve hypoplasia. Concomitant strabismus occurred in 13. Other abnormalities included microphthalmos, 
coloboma, cataract, and nystagmus. Mental retardation required special school placement for 16, and only 3 attended normal schools without extra assistance. The finding of ocular abnormalities in a child suspected of having FAS should strengthen the diagnosis.

Developmental neurotoxicity of PCBs in humans is reviewed from the Institute of Environmental studies, University of Illinois at UrbanaChampaign, Urbana, IL. (Schantz SL. Neurotoxicol and Teratology May/June 1996;18:217-227). Studies included those in Yusho, Japan; Yucheng, Taiwan; Michigan; North Carolina; Oswego, NY; New Bedford, MA; on Inuit people in the Arctic regions of Quebec; and in Faroe Islanders. Concurrent methylmercury poisoning may be an issue in interpretation of some studies. Children born to mothers exposed to PCBs showed abnormalities in behavior and development, including higher activity levels, behavior problems, lower IQ scores, decreased birth weight and head circumference, lowered scores on the Brazelton Neonatal Battery, deficits in memory at 4 years, and delays in psychomotor development. Although the deficits in cognition were often small, the public health implications of low-level PCB exposure was compared to that of lead exposure. At a population level, a decrease of 4 points on the Bayley Scales is estimated to result in a $50 \%$ increase in the number of children with subnormal scores. Subtle alterations in neuropsychological functioning caused by exposure to these environmental toxins were proposed as explanations for some cases of ADHD, either by a direct effect on the brain in the prenatal period or secondary to effects on thyroid function. The potential impact of postnatal exposure to PCBs via breast milk was also reviewed.

Maternal smoking is a preventable cause of mental retardation according to a study at the Rollins School of Public Health of Emory University, Atlanta, GA. (Drews CD et al. Pediatrics April 1996;97:547553). Children whose mothers smoked one pack a day during pregnancy had more than a $75 \%$ increase in mental retardation. Maternal smoking has also been linked to lesser impairments of cognitive function and academic achievement, auditory deficits, and behavioral problems in children.

Environmental factors should be considered more frequently as potential causes of attention deficits and learning disabilities.

\section{CNS IRRADIATION EFFECTS ON PUBERTY AND GROWTH}

The relation between age at irradiation, sex, and age at puberty onset was determined in 36 children treated with high-dose irradiation for tumors anatomically distant from the hypothalamic-pituitary region at the New York University Medical Center, St Luke's/Roosevelt Hospital, and Memorial Sloan Kettering Cancer Center, New York. Twenty six also received chemotherapy. Age at onset of puberty and pubarche was significantly earlier in girls but not in boys. The mean age at onset of puberty in both boys and girls was linearly correlated with age at diagnosis and irradiation, and was inversely related to body weight. (Oberfield SE et al. Age at onset of puberty following high-dose central nervous system radiation therapy. Arch Pediatr Adolesc Med June 1996;150:589-592). (Reprints: Sharon E Oberfield MD, New York University Medical Center, 550 First Ave, New York, NY 10016).

COMMENT. High dose cranial irradiation may cause precocious puberty in girls, particularly in those who are overweight. 